\title{
Low Level Flight Monitoring - High performance graphics
}

\author{
A. Rodrigo López Parra ${ }^{1}$ \\ ${ }^{1}$ Airbus Defence and Space, Sevilla - Spain \\ rodrigo.I.lopez@airbus.com
}

\begin{abstract}
:
Low level flight trials in the A400M require special ad-hoc software with high performance graphic for monitoring and analysis in real time, able to provide insight and analysis as quickly as possible, given the criticality of the maneuvers and the short response time available.

This software provides graphic views in back, side and plan. Showing behaviour and predicting aircraft maneuvers in autopilot mode very close of the terrain, between the mountains.

As well as being able to implements the analysis of tactical functionalities such as, reconnaissance, proximity and visualization of the terrain, guided and planned trajectory for the autopilot, lateral and vertical deviations through safety corridors, dynamic scales of zoom and navigation modes, calculation of maximum performances in real time, compare results for different passes time, generate analysis result already completed, and much more ...
\end{abstract}

Key words: monitoring, high performance, autopilot, simulate, low level fligh, analysis

\section{Introduction}

Normally, to follow the tests dedicated to specific tasks, software screens are developed for monitoring the systems on which we want to carry out the test. The most common thing is to design screens for the control of temperatures, pressures, valves, powers, levels, etc ...

They tend to be quite standard and do not consume many graphic resources.

\section{Low level flight tests}

However, low level flights in auto-pilot, are one of the most complicated functionalities developed to date in a military aircraft. It is about flying in autopilot at speeds of up to 300 kts at a minimum altitude of $500 \mathrm{ft}$ above the ground, in mountainous terrain. avoid the defensive ground missile systems, as well as the ground fire.

In order to guarantee the safety of the crew on the test flights, monitoring software has been made for an analysis of the entire maneuver with a high level of detail. Which has required high requirements graphic.

It must be take into account that the flight engineer in charge of the test does not have access to the CDS (Control and Display System) of the pilots.

Therefore, it is necessary to provide a lot of navigation information with an intuitive, smooth, fast and real-time animation. Each one of the maneuvers, each one of the turns, each climb or descent, is critical and there is very short response time for any unforeseen or correction.

The objective is to reach the hostile zone without being detected by the radars and to

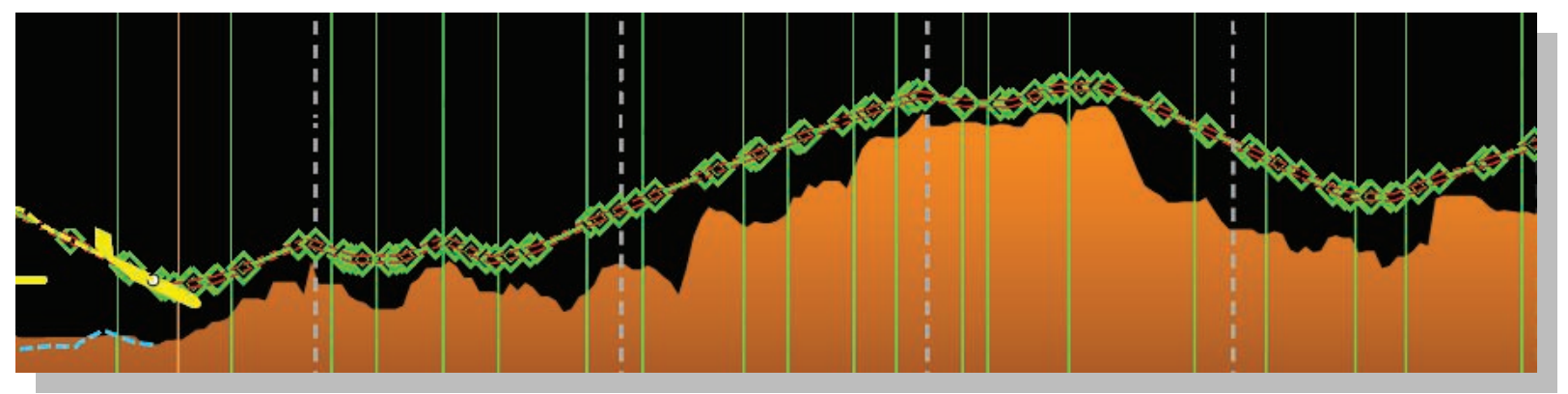

Figure 1. Example Profile of LLF 


\section{Design}

This ad-hoc software has been divided into three different views of the airplane; plan, side and back. With its position on the terrain, the path to follow and the safety corridors. In addition, another area of performance data with maximum slopes and navigation data has been defined. That tries to collect the limitations of the aircraft for maximum safety conditions.

The software allows us to change and compare between the information provided by the duplicated systems dedicated to calculate the trajectories.

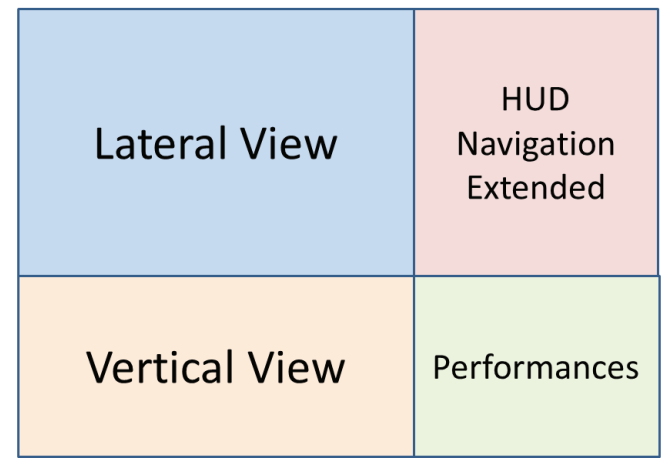

Figure 2. Graphic scheme

The software performs the reconstruction of the trajectories in specific data structures, similar to those of the aircraft navigation equipment, with the aim of making a detailed comparison with the trajectory shown by the aircraft.

\section{Trajectory}

If we want to define a trajectory, we need a geographic point of entry, a geographic point of exit, a minimum altitude referenced to the terrain and a speed.

These input data allow the systems to analyze the terrain to fly over and give us two trajectories, a lateral one and a vertical one. Since we move in a three dimensional system.

\section{Plan (Lateral View)}

It is a view of the aircraft followed from the sky called lateral view.

The lateral trajectory that the aircraft must follow is shown, defined as a set of segments that they can be straight or curved between geographic coordinates and between welldefined waypoints.

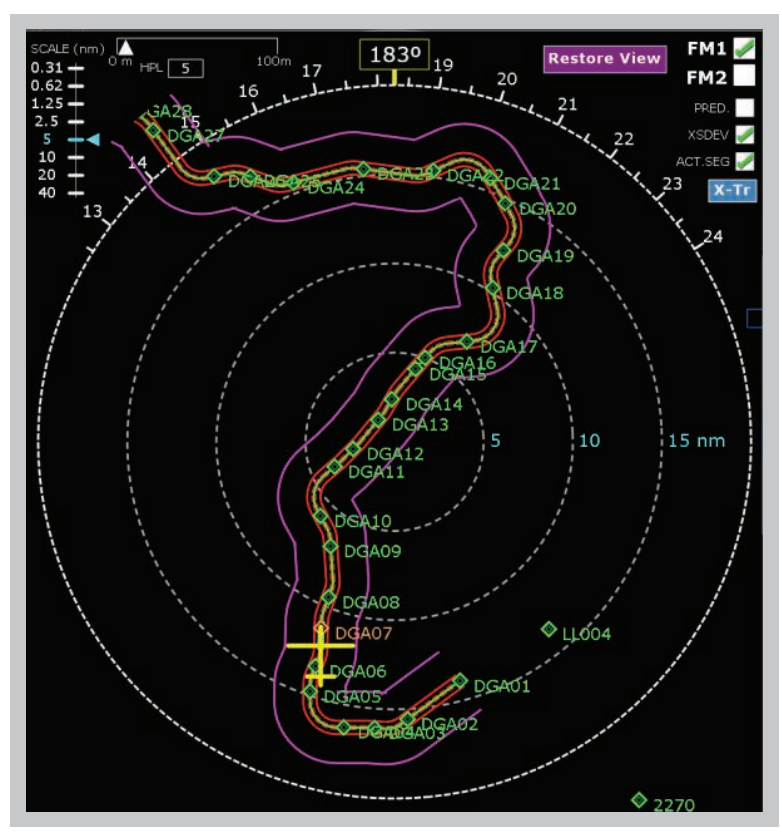

Figure 3. Complete lateral path

We can modify the zoom of distance scales just like the pilot would do in the PFD (Primary Flight Dislay), in fact we have the same scales as the aircraft itself.

We can explore the complete path and move freely in any direction. In this way, we can see whether the path is right or not in advance.

With smaller zoom levels we can note more useful elements.

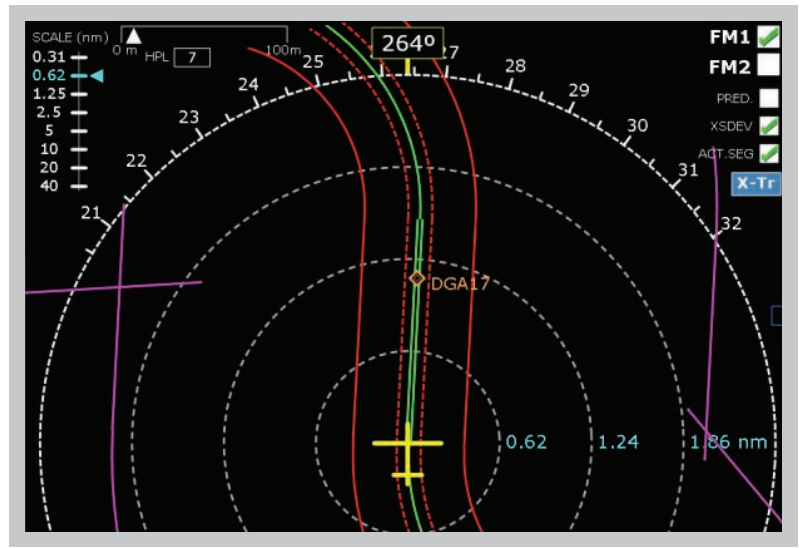

Figure 4. Lateral View

The autopilot must follow exactly the trajectory drawn in green that has been calculated by the systems that analyze the terrain, as the ideal.

To help detect any problem other features have also been developed.

The prediction of the airplane's turn is shown, in the same way that in the aircraft. 
The active segment of the trajectory located the aircraft by the system.

The active waypoint to which we are going.

The maximum lateral deviation referenced to the trajectory is shown too.

Three corridor are calculate and drawing from the given trajectory:

- Excessive desviation corridor.

There is some problem to keep the aircraft in the path of the trajectory.

- Safety corridor.

While we are inside this corridor, we will be safe.

- Safety altitude corridor.

It's related to safety altitude.

The safety altitude corridor is drawn for the evasion maneuver.

Also, we can compare the differences in the position of the aircraft between a high precision DGPS and the aircraft GPS.

\section{Back (Navigation Flight Display)}

This is the view that provides to the flight engineer more navigation information, since it reproduces the complete HUD of the aircraft, the navigation modes and monitors the errors that the systems can notify when building the trajectories.

It is possible, for example, that a LLF trajectory is required at a certain speed and that the system that calculate the trajectory notifies that it is not possible to perform it due to an impossible turn or climb for the aircraft's performance.

In addition, the safety graphic $U$ is added. It is a corridor seen from a back of the plane, and of which, the mock-up of the plane should never leave to be safe.

It emulates the presentation of the plane's instruments so that they feel familiar, but all geometric calculations are done internally in real time.

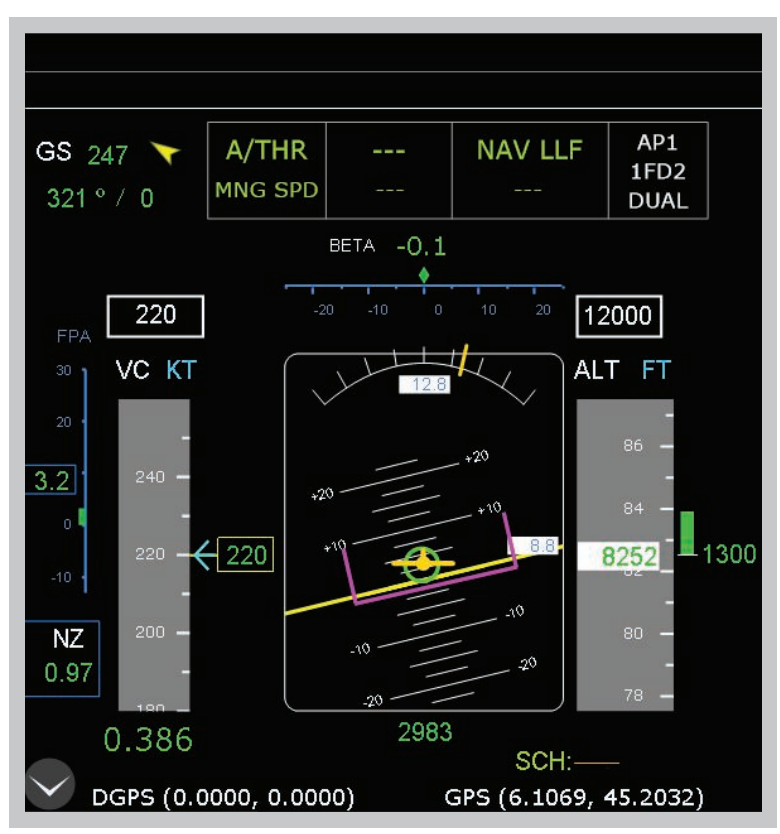

Figure 5. HUD Extended

\section{Performance}

This zone collects information of the maximum slope that the aircraft can perform, and the slopes that have the following segments to fly.

The autopilot systems must have verified that all the maneuvers of the LLF path are possible to do, but it never knows, better to check it again.

For the calculation of the performances, the failure of motors can be taken into account for maximum safety.

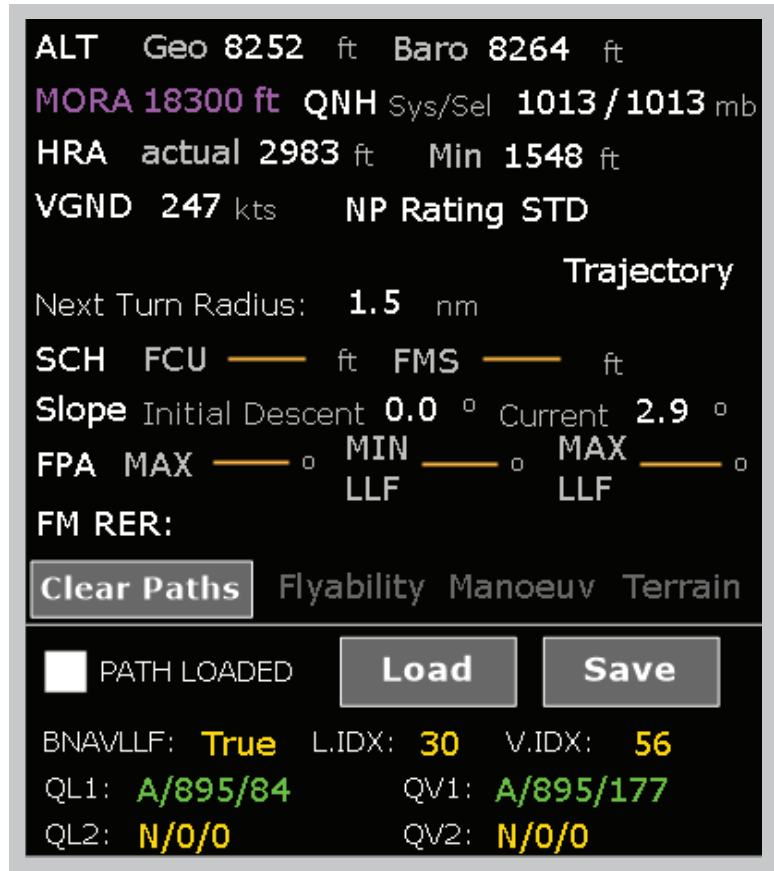

Figure 6. Performances 


\section{Side (Vertical View)}

This view is the most important of the system, it shows the plane and the terrain from its profile, matching at all times, what is the altitude of the aircraft with respect to the terrain, as well as the trajectory that the autopilot will perform.
At the same time, the path followed by the plane in altitude and radio altimeter is shown and recorded, so you can have a complete trace of the entire aircraft path.

For a vision at all levels we can zoom scales in altitude and distances, apart from moving freely throughout the flight profile.

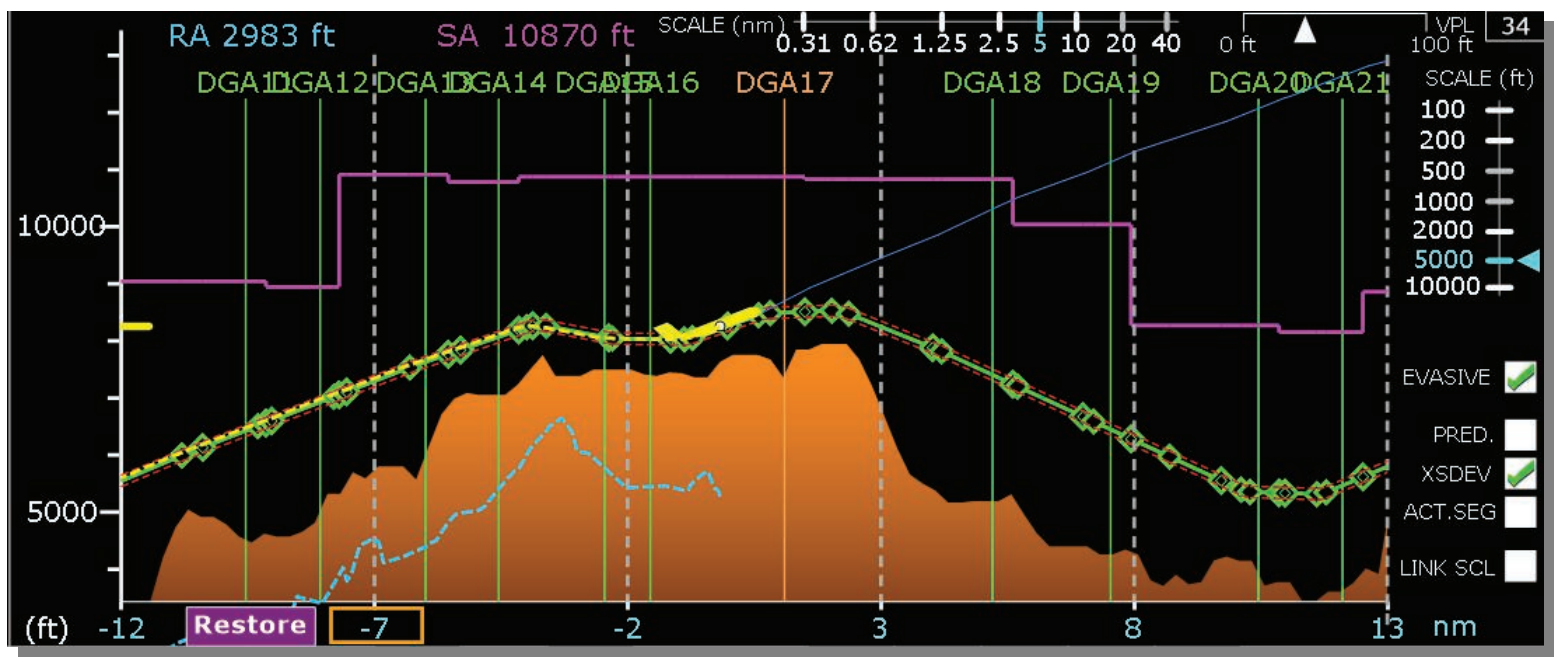

Figure 7. Vertical View

The profile is built as if we were passing a rope for each geographical point that the aircraft is going to fly and we extend it until it is straight. Getting the altitude at each point of that rope.

We can see the A/C mockup how it changes in the climbs and descents through the profile of the mountainous terrain.

The vertical trajectory that the aircraft must follow is defined by a set of straight and curved segments referenced to altitudes and geographic coordinates through the lateral segments of the lateral trajectory.

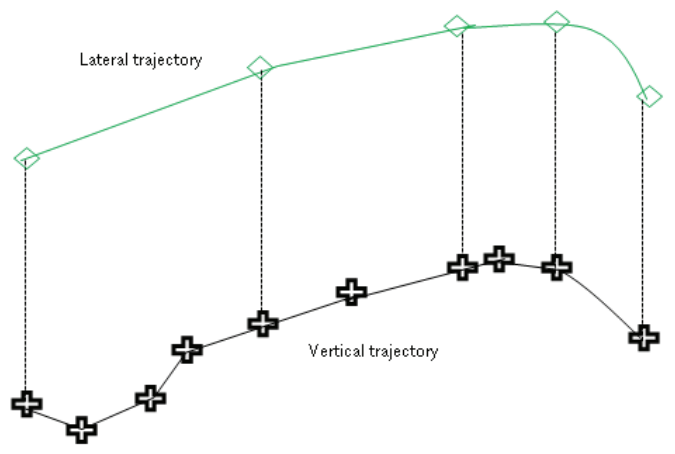

Figure 8. Vertical and lateral segment distribution

We identify the position of the aircraft, reference it to the geographical waypoints, the abscissa of the vertical segments and the exit point of the trajectory.
The vertical predictor of aircraft climb is provided (information extended to the airplane itself).

Starting from the vertical trajectory, we draw the corridor of maximum deviation in altitude and the corridor of safety altitude for the evasive trajectory in case of emergency.

This evasive path is also possible to show at the request of the flight engineer.

In case of any problem following the trajectory, the aircraft will abort the LLF route and perform the evasion maneuver until reaching a safe altitude.

The software automatically saves all the sessions flown in files so that at any time you can display a comparison view with a previous session.

The same session flown on different days will surely give slightly different results if there is no problem. Due to differences in climatic conditions, changes in aircraft performance occur. 


\section{Rebuilding of the terrain, decoding busses of aircraft}

The process to get the terrain has been a new challenge for this software to be really useful. Everything is referenced to this terrain in the vertical view and the most important thing is to know how close the airplane is from the terrain, as we can imagine.

It has had to perform an exclusive research to decode protocol data broadcasted on aircraft busses.

In this way, a lot of valuable information has been obtained (which is not even available for the airplane itself) in order to carry out this software.

The processes have been optimized to avoid the loss of any type of data related to any of the equipment that works in the aircraft autopilot system.

\section{Analysis in real time}

The vision and data provided by this software allows a complete analysis and diagnosis of this complicated system, just after the flight.

This software has also been integrated into test benches and simulators, given the enormous support it gives even to preliminary tests on real flights.

\section{Safety critical to Test}

Being a very complex system and still in development, we must be very careful to the behavior of the systems that are being tested. With this software, you can know how the aircraft will act before it does and anticipate if there is a problem in the definition of the trajectory and/or its route.

The changes with each new standard software in LLF equipment are frequent and this software is in continuous improvement.

\section{References}

[1] German Gallego Garcia, RTF Low Level Flight A400M, 2016 - 2018. 